process. Apatite-like nanoparticles were observed on the MBG-PCL surface after soaking for $4 \mathrm{~h}$, and soaking for $24 \mathrm{~h}$ resulted in a MBG-PCL fully covered with apatite. Likewise, energy dispersive x-ray (EDX) analysis gave the same result. The biocompatibility of the MBG-PCL was evaluated by MTT [3-(4,5-Dimethylthiazol-z-YI)-2,5-Diphenyltetrazolium Bromide] assay. The cell viability on the MBG-PCL surface was found to be significantly larger than that on the PCL surface. These studies demonstrate excellent bioactivity and biocompatibility of this MBGPCL scaffold, the researchers said.

The method developed in this work was used to fabricate a scaffold for tissue engineering. However, by utilizing alternative materials, the researchers showed that this method can be used in "applications involving biomedical devices, drug delivery systems, filters, catalysis, and optics."

CHANG ZHONG

\section{Atomic Force Microscopy Used to Detect Cancer Cells}

Immunohistochemical and cytomorphological analyses are currently used to detect cancer cells, but morphological overlap between tumor and normal cell types often poses problems for these techniques. In the past few years, however, an increase in cell elasticity has been recognized as a marker for disease and associated with cell adhesion and cytoskeletal organization. S.E. Cross and J.K. Gimzewski at the California NanoSystems Institute and the University of California, Los Angeles, and Y.-S. Jin and J. Rao at the University of California, Los Angeles used atomic force microscopy (AFM) to distinguish cancerous cells from normal cells.

As reported in a letter published in the December 2, 2007 issue of Nature Nanotechnology (p. 780; DOI:10.1038/nnano. 2007.388), the researchers used standard protocols to collect from patients with suspected metastatic adenocarcinoma samples of malignant and benign mesothelial cells in pleural effusions (adenocarcinoma is a cancer that originates in glandular tissue; mesothelial cells form part of the membranes covering body cavities, and an effusion is an abnormally large collection of fluid in the space surrounding the lungs). Ex vivo growth during a 12-h incubation period differentiated normal cells, which have a large, flat morphology, from benign cells, which exhibit anchorage-resistant morphology such as rounding. For each cell, elasticity was quantified as Young's modulus $E$ from force-displacement curves recorded from AFM performed at a rate of $1 \mathrm{~Hz}$ at $37^{\circ} \mathrm{C}$. Using samples from seven patients, average $E$ values of $0.53 \pm$
$0.10 \mathrm{kPa}$ and $1.97 \pm 0.70 \mathrm{kPa}$ were obtained for 40 malignant and 48 benign mesothelial cells, respectively. Similar average $E$ values were found for samples from a single patient, showing that the cell stiffness of metastatic cancer cells is about $73 \pm 11 \%$ less than benign mesothelial cells. Malignant cells displayed a narrow distribution of $E$ values while the benign cells displayed a broad peak.

For one particular clinical sample consisting of cells that were difficult to classify as either benign or malignant, ex vivo culturing resulted in two populations of cells with different morphologies. However, nanomechanical analysis based on AFM measurements determined that both populations were malignant cells, which was confirmed with immunohistochemical staining. The researchers said that "the correlation of cytomechanical measurements with immunohistochemical analysis suggests that nanomechanical measurements of cancer cells has potential for the detection of cancer and may aid in personalized selection of medication and drug screening, especially in body cavity effusions where accurate diagnosis based solely on morphology has to date been challenging." STEVEN TROHALAKI

\section{Embedded Capacitive Circuit Detects, Characterizes, and Manipulates Droplets in Microfluidic Systems}

Droplets in microfluidic systems are presently detected using optical devices, such as single element photodiodes for counting or high-speed charge-coupled device image sensors with imageprocessing software for imaging. A group of researchers from The Hong Kong University of Science and Technology reported in the October-December 2007 issue of Biomicrofluidics (DOI: $10.1063 / 1.2795392)$ on a novel method for real-time detecting, characterizing, and controlling of such droplets through the use of capacitive sensors; the dielectric constant contrast between the fluid of interest and the carrying fluid is the only prerequisite for the method functionality.

The research group, including $X$. Niu, M. Zhang, S. Peng, W. Wen, and P. Sheng, designed a microfluidic chip that embeds from the fabrication process (using soft lithography) a series of parallel electrodes placed across the microfluidic channel, some of which are connected to a capacitance resonance detection system and others to a square wave signal generator for in situ manipulation of the droplets.

The researchers were able to calibrate the device for measurements involving length (and subsequently volume) of nano- and picoliter range aqueous droplets in silicone oil by analyzing the resonance circuit voltage changes as the droplets were passing through the electrodes. Very good accuracy was obtained for droplet lengths bigger than half of the electrode width, and a detection frequency of more than $1 \mathrm{kHz}$ (1100 droplets per second) has been attained, limited in this case only by the sustainable pressure of the chip and not by the detection system. The droplets velocity was also easily calculated by measuring the time required to reach from one set of electrodes to the other.

The system was subsequently calibrated and employed to detect droplets of different composition (ethylene glycol and, respectively, water) and to sort them in two different branches of a T-shaped microchannel using a feedback system that employs a set of electrodes with a positive high potential to charge the droplets and another set that can preferentially change polarity to direct the droplets through either of the branches. The delay time of the feedback system was adjusted for the droplet's velocity so that a perfect match was obtained between detection and manipulation.

The researchers demonstrated that their method of real-time labeling, sorting, and manipulation of droplets can provide a very simple and cost-effective alternative for portable devices involved in biomicrofluidic processing, digital microfluidics, microchemical reactions, and environmental monitoring.

EUgEN PANAITESCU

\section{Quasi-Forbidden Bragg Peaks Demonstrated as Inherent Property in Homogeneous Ordered Soft Materials}

Bragg peaks exist in highly ordered soft materials that cannot be indexed assuming homogeneous crystal structures and that have been attributed to changes in the crystal structure induced by the ordering process. S. Förster and co-workers from the University of Hamburg, Germany, together with S.V. Roth from DESY, Germany and P. Lindner from the Institute Laue-Langevin, France, have demonstrated that these unexpected Bragg peaks are an inherent property of homogeneous ordered soft materials and arise from the spontaneous ordering allowed by the weak interaction potential that tolerates imperfections of the constituent structures that limit the coherence of the crystalline lattice. Their results have been reported in the November 2007 issue of Nature Materials (p. 888; DOI: 10.1038/ nmat1995). "This explained the presence of quasi-forbidden Bragg peaks in lyotrop- 